\title{
Diamond like carbon coatings deposited by microwave plasma CVD: XPS and ellipsometric studies
}

\author{
R M DEY*, M PANDEY ${ }^{\dagger}$, D BHATTACHARYYA ${ }^{\dagger \dagger}$, D S PATIL ${ }^{\dagger \dagger}$ and S K KULKARNI \\ Department of Physics, University of Pune, Pune 411 007, India \\ ${ }^{\dagger}$ Synchrotron Radiation Section, ${ }^{\dagger \dagger}$ Spectroscopy Division, ${ }^{\dagger \dagger \dagger}$ Laser and Plasma Technology Division, \\ Bhabha Atomic Research Centre, Mumbai 400 085, India
}

MS received 3 May 2007

\begin{abstract}
Diamond-like carbon (DLC) films were deposited by microwave assisted chemical vapour deposition system using d.c. bias voltage ranging from $-100 \mathrm{~V}$ to $-300 \mathrm{~V}$. These films were characterized by $\mathrm{X}$-ray photoelectron spectroscopy (XPS) and spectroscopic ellipsometry techniques for estimating $s p^{3} / s p^{2}$ ratio. The $s p^{3} / s p^{2}$ ratio obtained by XPS is found to have an opposite trend to that obtained by spectroscopic ellipsometry. These results are explained using sub-plantation picture of DLC growth. Our results clearly indicate that the film is composed of two different layers, having entirely different properties in terms of void percentage and $s p^{3} / s p^{2}$ ratio. The upper layer is relatively thinner as compared to the bottom layer.
\end{abstract}

Keywords. Diamond like carbon films; microwave assisted CVD; X-ray photoelectron spectroscopy; spectroscopic ellipsometry.

\section{Introduction}

Diamond like carbon (DLC) films have received a great deal of attention in industries due to their various excellent properties related to high hardness and wide optical gap (Angus and Hayman 1988). Their chemical inertness makes them suitable in wear resistance tools in highly corrosive atmospheres. DLC coatings are known to have good biocompatibility (Linder et al 2002) and have found a wide range of interesting medical applications (Grill 2003). The properties of these films are mainly determined by the $s p^{3} / s p^{2}$ ratio which can be tailored by deposition conditions. While high value of $s p^{3} / s p^{2}$ ratio makes the films closer to that of diamond, their low value makes them closer to graphite. The electronic, optical and structural properties of DLC films strongly depend on the delicate balance between $s p^{3}$ and $s p^{2}$ hybridization of the carbon atoms and the amount of hydrogen present in these films (Robertson 1994). The determination of $s p^{3} / s p^{2}$ ratio in these films is, therefore, quite important. Interestingly the $s p^{3} / s p^{2}$ ratio can vary along the growth direction as revealed by some simulation studies by Uhlmann et al (1998). Hereby using the complementary techniques such as X-ray photoelectron spectroscopy (XPS) probing thin upper layers $(\sim$ few $\mathrm{nm})$ and spectroscopic ellipsometry probing layers beneath $\sim$ a few micrometers, we

*Author for correspondence (ravi@physics.unipune.ernet.in) can indirectly show the experimental evidence for the same.

A large number of growth techniques like mass selected ion beam deposition (Savvidas 1986), pulsed laser deposition (Martin et al 1988), rf and d.c. magnetron sputtering (Martinu et al 1992, 1993), filtered cathodic vacuum arc (Kutsay et al 2003), electron cyclotron resonance (ECR) plasma CVD (Weissmantel et al 1980), and microwave plasma CVD (MPCVD) (Patil et al 1997) have been used for DLC deposition. However, the most important factor applicable in almost each of the above methods, which dictates the growth of DLC films, is the energy of impinging carbon ions and the neutrals in the growing film. The energy spread of carbon ions and neutral atoms at the moment of impact is sufficiently wide so that the coating grows with a mixture of $s p^{3}$ and $s p^{2}$ bonding. The bias voltage is found to play an important role in determining the $s p^{3} / s p^{2}$ ratio in the present DLC films. While XPS is suited mostly to the top surface layers, ellipsometry provides bulk information of the DLC coating. The studies carried out using both the techniques, therefore, provide a comprehensive picture of DLC films grown under present conditions. In this paper, we present our investigations of DLC coatings prepared under microwave chemical vapour deposition using X-ray photoelectron spectroscopy (XPS). The $s p^{3} / s p^{2}$ ratio estimated from spectroscopic ellipsometry and XPS techniques are found to show different trends with bias voltage. These results are best explained using sub-plantation model described by Lifshitz (Kutsay et al 2003) and thermal spike model by Weissmantel et al (1980). 


\section{Experimental}

A microwave plasma processing facility, having a magnetron source operating at $2.45 \mathrm{GHz}$ with varying power, was used for growing DLC films. The description of the microwave system is given elsewhere (Patil et al 1997). The plasma chamber, which included a quartz tube, was evacuated to an initial pressure of $10^{-5}$ torr by a vacuum pumping system consisting of a rotary and a diffusion pump. Microwave plasma was generated using argon as a carrier gas $(10 \mathrm{sccm})$ by passing 150 watts of microwave power. After stabilization of the plasma, methane as a precursor gas $(3 \mathrm{sccm})$ was introduced along with the argon gas into the plasma chamber. The final deposition was carried out at a pressure of $5 \times 10^{-2}$ torr for $\sim 1 \mathrm{~h}$ on silicon substrates.

Prior to deposition, the substrates were ultrasonically cleaned with isopropyl alcohol, and then inserted into the plasma chamber. Uniform plasma was generated over the substrate area, and a d.c. bias which varied from $-100 \mathrm{~V}$ to $-300 \mathrm{~V}$ was applied to the substrate. Films of good optical quality were produced. The electrical resistivity of these films was higher than $200 \mathrm{M} \Omega$ indicating insulating behaviour. Average thickness of the films as determined from the profilometer and the ellipsometer was $\sim 1 \mu \mathrm{m}$.

Raman studies were carried out on different negative biased samples for confirmation of DLC formation.

$\mathrm{X}$-ray photoelectron spectroscopic studies were carried out using ESCALAB MKII spectrometer (VG scientific, $\mathrm{UK}$ ). Al-K $\mathrm{K}_{\alpha}$ (energy, $\sim 1486.6 \mathrm{eV}$ ) was used as incident $\mathrm{X}$-ray radiation and concentric hemispherical energy analyser operating with $50 \mathrm{eV}$ pass energy was used for energy analysis of the photoelectrons. This gave an overall spectral resolution of $\sim 1 \mathrm{eV}$ for photoelectron peaks.
Pyrolytic graphite was used as a reference for the spectra. The pressure in the analysing chamber was $\sim 10^{-8}$ torr. Prior to XPS studies, DLC samples were ultrasonically cleaned using isopropyl alcohol, and then dried to avoid any contamination and then were inserted in the spectrometer. The spectroscopic ellipsometric studies were carried out using a spectroscopic phase modulated ellipsometer (model UVSEL ${ }^{\mathrm{TM}} 460$, ISA JOBIN-YVON, SPEX) in the range $300-1000 \mathrm{~nm}$.

\section{Results and discussion}

Raman spectrum as shown in figure 1 of all the samples biased at different voltages $(-100 \mathrm{~V}$ to $-300 \mathrm{~V})$ shows two prominent peaks centred approximately at $1332 \mathrm{~cm}^{-1}$ ( $D$ band) and $1550 \mathrm{~cm}^{-1}$ ( $G$-band) indicating DLC formation (Ferrari and Robertson 2000).

DLC films with high $s p^{3}$ content are always desirable to achieve properties closer to that of diamond. The energy spread in the plasma species of CVD process leads to DLC coatings with a mixture of $s p^{3}$ and $s p^{2}$ bonding. Bias voltage is found to play an important role in determining the nature of bonding in these films. The present work describes the studies of DLC films as a function of bias voltage.

Photoelectron survey scan was recorded from 0$1250 \mathrm{eV}$ binding energy, followed by a detailed carbon $1 \mathrm{~s}$ peak scan. Figure 2 shows the survey scan of a typical DLC film grown at a bias voltage of $-200 \mathrm{~V}$. The survey scan also shows a signal at $\sim 532 \mathrm{eV}$ due to chemisorbed oxygen species $(\mathrm{O} 1 s)$ on the surface of the film. Detailed $\mathrm{C} 1 s$ spectra of all the films were recorded in the binding energy range $275-300 \mathrm{eV}$ and fitted using a XPS peak 4.1
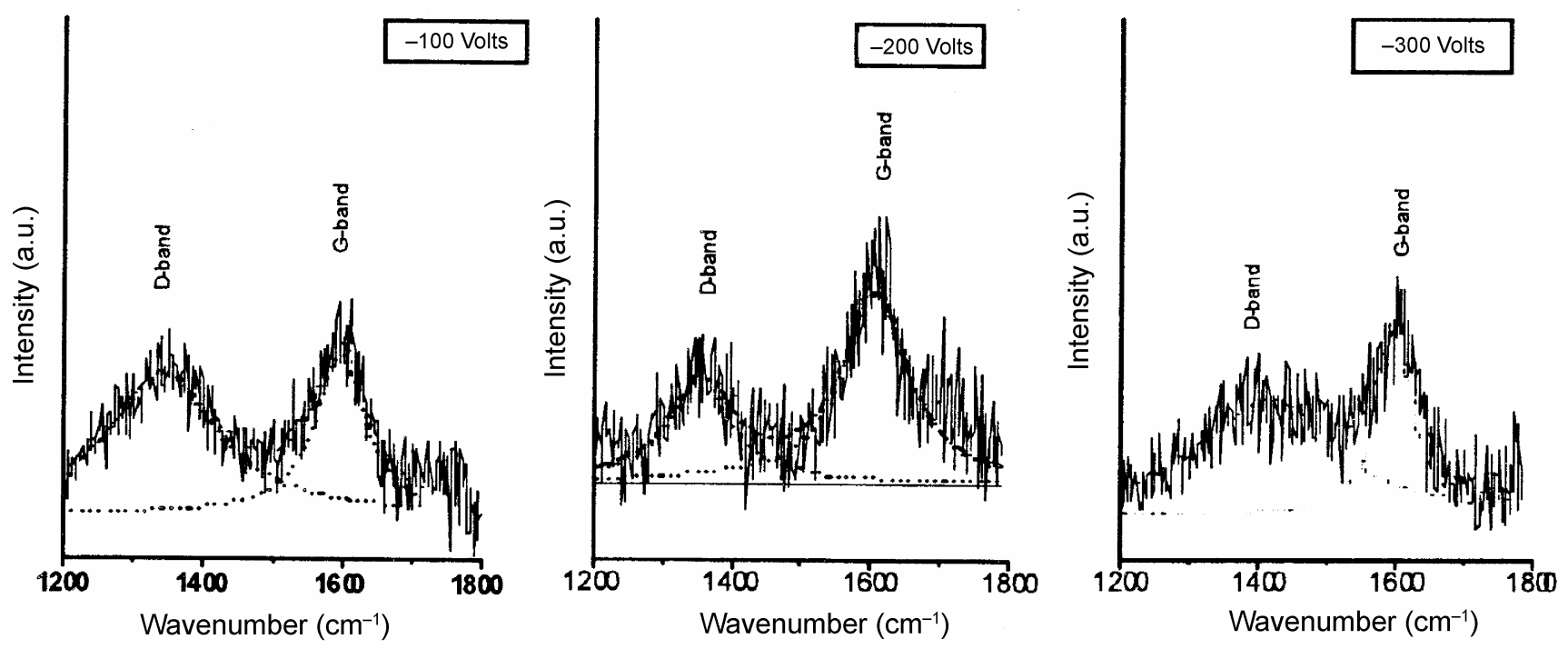

Figure 1. Raman spectrum of DLC samples grown at different negative bias voltages. 
Table 1. Details of the peak fitting analysis of $\mathrm{C} 1 s$ peak carried out by XPS studies.

\begin{tabular}{|c|c|c|c|c|c|c|}
\hline S1. no. & Bias & oltage (V) & $s p^{2}$ & $s p^{3}$ & $\mathrm{CO}$ & $s p^{3} / s p^{2}$ ratio \\
\hline 1. & -100 & $\begin{array}{l}\text { BE }(e V) \\
\text { FWHM }(e V)\end{array}$ & $\begin{array}{l}285 \\
2 \cdot 02\end{array}$ & $\begin{array}{r}286 \cdot 1 \\
2 \cdot 2\end{array}$ & $\begin{array}{r}288 \cdot 1 \\
2 \cdot 2\end{array}$ & $1 \cdot 70$ \\
\hline 2. & -200 & $\begin{array}{l}\mathrm{BE}(\mathrm{eV}) \\
\text { FWHM }(\mathrm{eV})\end{array}$ & $\begin{array}{l}285 \\
2 \cdot 02\end{array}$ & $\begin{array}{r}285 \cdot 9 \\
2 \cdot 2\end{array}$ & $\begin{array}{r}288 \cdot 0 \\
2 \cdot 0\end{array}$ & 1.57 \\
\hline 3. & -250 & $\begin{array}{l}\mathrm{BE}(\mathrm{eV}) \\
\text { FWHM }(\mathrm{eV})\end{array}$ & $\begin{array}{c}285 \\
1 \cdot 9\end{array}$ & $\begin{array}{r}285 \cdot 6 \\
2 \cdot 3\end{array}$ & $\begin{array}{r}287 \cdot 4 \\
2 \cdot 2\end{array}$ & $1 \cdot 21$ \\
\hline 4. & -300 & $\begin{array}{l}\mathrm{BE}(\mathrm{eV}) \\
\text { FWHM }(\mathrm{eV})\end{array}$ & $\begin{array}{l}285 \\
1.95\end{array}$ & $\begin{array}{r}285 \cdot 6 \\
2 \cdot 1\end{array}$ & $\begin{array}{r}287 \cdot 9 \\
2 \cdot 2\end{array}$ & 1.03 \\
\hline
\end{tabular}

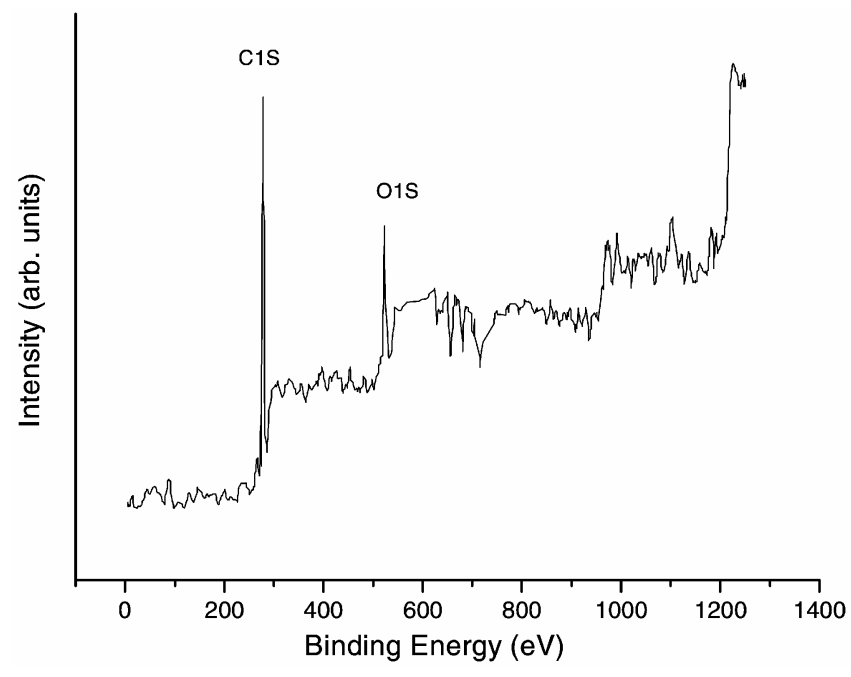

Figure 2. Survey scan of DLC film deposited at $-200 \mathrm{~V}$, showing peaks corresponding to $\mathrm{C} 1 s$ and $\mathrm{O} 1 s$.

software. Gaussian to Lorentzian ratio (G/L ratio) of $30: 70$ (obtained from the fitted peak of pyrolytic graphite), has been consistently used in our curve fitting. Figure 3 shows the fitted spectra for carbon $1 s$ of DLC film deposited at $-100 \mathrm{~V},-200 \mathrm{~V}$ and $-300 \mathrm{~V}$. C $1 s$ spectrum is fitted with three peaks corresponding to $s p^{3}$ carbon (diamond), $s p^{2}$ carbon (graphite) and CO (adsorbed gas). A small contribution from $\mathrm{CO}$ is found to be present in all samples, and may be related to the chemisorbed oxygen present in the DLC films. The fitted spectra match very well with the experimental data. Low observed value of the residuals show the validity of our fitting. The peak positions, their full width at half maxima (FWHM) and the $s p^{3} / s p^{2}$ ratio obtained from the area fraction of $s p^{3}$ peak and $s p^{2}$ peak in $\mathrm{C} 1 s$ spectrum are given in table 1 .

As can be seen from table 1 , the $s p^{3} / s p^{2}$ ratio of the films estimated from the peak fitting of $\mathrm{C} 1 s$ spectrum is found to decrease with increase in the bias voltage. In fact, this ratio varies only in the range 1.7 (at bias voltage, $\sim-100 \mathrm{~V}$ ) to 1 (at bias voltage, $\sim-300 \mathrm{~V}$ ). Interestingly this result has an opposite trend to that observed by our spectroscopic ellipsometric studies where the $s p^{3} / s p^{2}$ ratio varies from 5.4 (at bias voltage, $\sim-100 \mathrm{~V}$ ) to 13.6 (at bias voltage, $\sim-300 \mathrm{~V})$. The variation in the $s p^{3} / s p^{2}$ ratio as a function of bias voltage as obtained by XPS and ellipsometry is schematically shown in figure 4 . Although $\psi$ (the amplitude ratio of the two mutually perpendicular polarization components) and $\Delta$ (the phase difference between these two components) are the only observables in spectroscopic ellipsometry, a proper analysis can provide a large information related to percentage of voids, band gap, band tails and $s p^{3} / s p^{2}$ ratio. In fact, the measured ellipsometric spectra are fitted with an appropriate Firouhi and Blommer (1986) model assuming a realistic sample structure where the layer thickness and optical constants are used as the fitting parameters. The optical constants of the substrates have been supplied and the trial dispersion relations have been used for the films. As is true in our case, for a composite layer structure (consisting of more than one material, or material(s) and void), the calculation for the effective optical constants have been done using Bruggeman (1935) effective medium approximation (EMA) model, which describes a composite of aggregated phases or random mixture microstructure. A two-layer composite structure on a silicon substrate (percentage of voids being different for each layer) is found to provide the best fit for all of our samples. Further increase in the number of layers does not improve the quality of fitting. Details for obtaining these parameters are described elsewhere (Pandey et al 2004). Our earlier ellipsometric results (Bruggeman 1935) have already suggested a composite structure of DLC film consisting of two distinct layers. The top layer has a large percentage of voids and the bottom layer has less percentage of voids. In addition, the latter is relatively thicker than the former. Another interesting thing to note is that as the bias voltage is increased the percentage of voids in both the layers is reduced. This effect is quite prominently observed in case of DLC film grown at $-300 \mathrm{~V}$. The $s p^{3} / s p^{2}$ ratio is found to increase with increase in bias voltage.

As shown in figure 4 , while spectroscopic ellipsometry suggests an increase in $s p^{3} / s p^{2}$ ratio with increase in bias voltage, XPS studies show small but definite variation which is a decreasing trend in the $s p^{3} / s p^{2}$ ratio as a function of bias voltage. It may be noted that while the measurements carried out by XPS provide information pertaining to top few mono-layers, the spectroscopic ellipsometry gives 

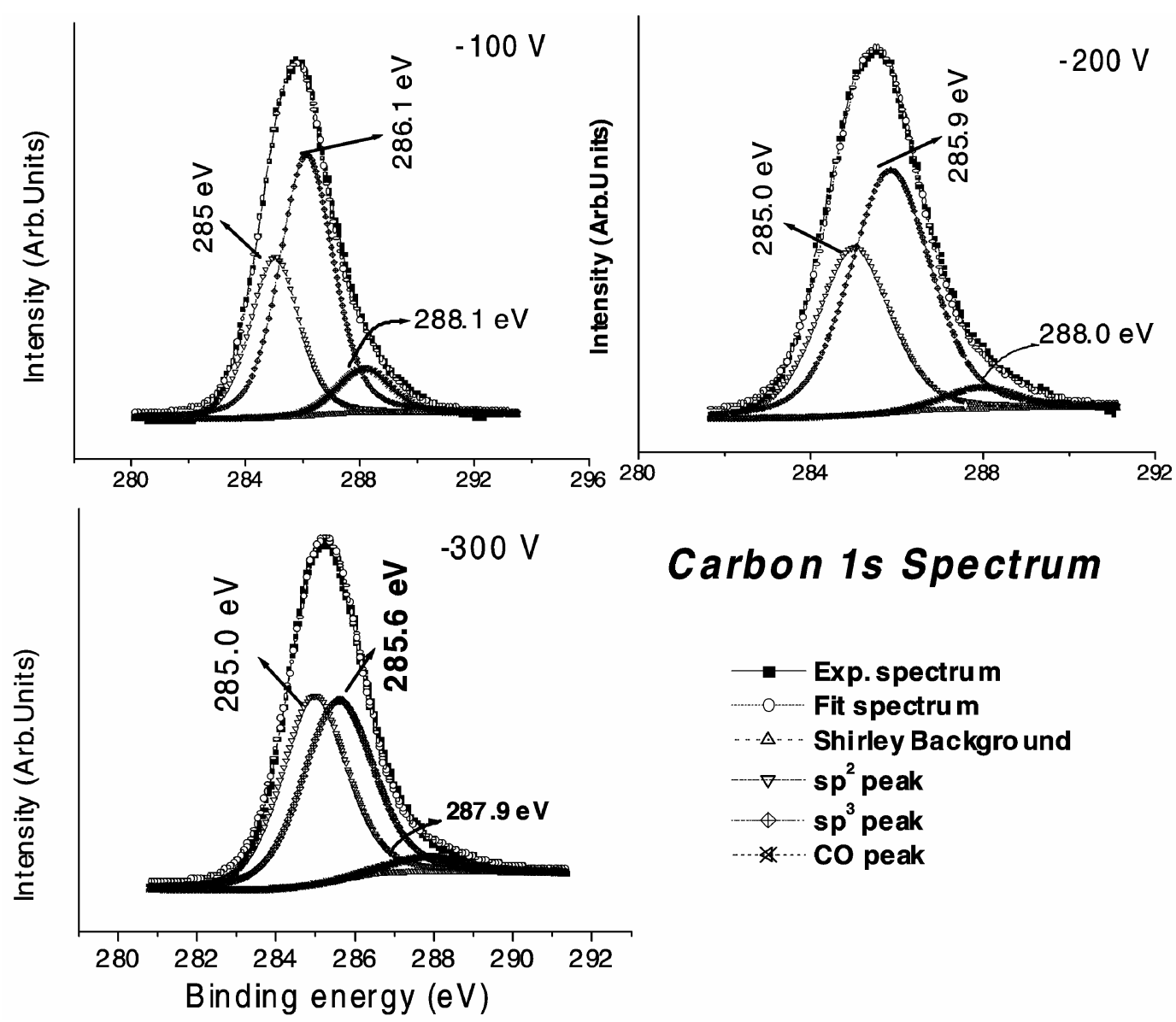

Carbon 1s Spectrum

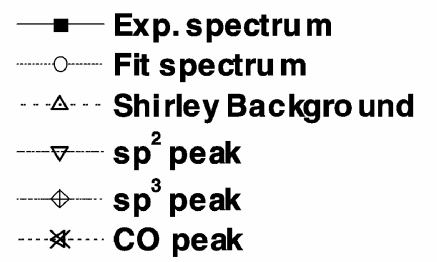

Figure 3. Fitted C1s spectra of DLC film deposited at $-100 \mathrm{~V},-200 \mathrm{~V}$ and $-300 \mathrm{~V}$, indicating peaks corresponding to $s p^{3}-\mathrm{C}, s p^{2}-\mathrm{C}$ and $\mathrm{CO}$.

Table 2. Details of the spectroscopic ellipsometry studies.

\begin{tabular}{lcccccc}
\hline Sample no. & Bias voltage (V) & $s p^{3} / s p^{2}$ ratio & $\begin{array}{c}\text { Layer 1 } \\
\text { thickness }(\AA)\end{array}$ & Voids (\%) & $\begin{array}{c}\text { Layer 2 } \\
\text { thickness }(\AA)\end{array}$ & Voids (\%) \\
\hline DLC13 & -100 & $5 \cdot 38$ & 614 & $40 \cdot 9$ & 15955 & $33 \cdot 0$ \\
DLC14 & -150 & $6 \cdot 25$ & 336 & $46 \cdot 6$ & 7099 & $42 \cdot 1$ \\
DLC12 & -200 & $8 \cdot 80$ & $693 \cdot 3$ & $40 \cdot 0$ & 17088 & $30 \cdot 6$ \\
DLC16 & -300 & $13 \cdot 58$ & 588 & $31 \cdot 6$ & 18865 & $18 \cdot 3$ \\
\hline
\end{tabular}

the overall contribution from a larger depth beneath. This is in good agreement with the studies carried out by Filik et al (2003), where they have suggested the $s p^{3} / s p^{2}$ ratio estimated by XPS to be entirely different from that in the bulk. The apparent differences in the results obtained by XPS and ellipsometry may, however, be understood with sub-plantation model described by Lifshitz (Kutsay et al 2003) and Uhlmann et al (1998) as well as thermal spike models by Weissmantel et al (1980). Sub-plantation model provides the most successful picture of DLC growth process. According to the subplantation model the energetic bombardment of carbon ions and neutral atoms present in
CVD plasma on the growing film results in the formation of a defective layer commonly referred to as a sub-surface layer. It is low in density and rich in $s p^{2}$ content. The $s p^{3}$ content in this layer is expected to vary with bias voltage and would be lower at higher bias voltage. Below this layer lies an $s p^{3}$ rich matrix with high density. Figure 5 shows schematic representation of a thin sub-surface layer, below which lies the bulk of the DLC having larger thickness. It needs to be mentioned that the XPS is able to probe only this thin sub-surface layer, while the spectroscopic ellipsometry probes the entire DLC film i.e. sub-surface as well as the layers beneath. Uhlmann et al 
(1998) carried out first principle calculations using density functional molecular dynamics studies of the events happening during the growth of DLC films. It appears that the collision cascades during the DLC growth result in a porous region near the surface having lower density. The deeper region consists of dense $s p^{3}$ fraction. This type of composite layer i.e. a layer with lower density followed by a layer with high density, is a consequence of a sub-plantation model. Successive deposition increases thickness of the interior layer, while the top layer remains as it is.

Another process i.e. the growth of DLC associated with the high pressure compression (13 GPa) (Weissmantel et al 1980), has also been reported in the literature. According to this process, the high temperature and the high pressure generates a thermal spike during compression which is sufficient for the $s p^{2}$ to $s p^{3}$ conversion. This process is, however, more dominant at higher bias voltage. Both the mechanisms i.e. incorporation of carbon species in the sub-surface region and the thermal spikes in the compression process, may together be responsible for the DLC growth. While the impingement energy of the incident ions have a more direct effect on the top layer, the interior

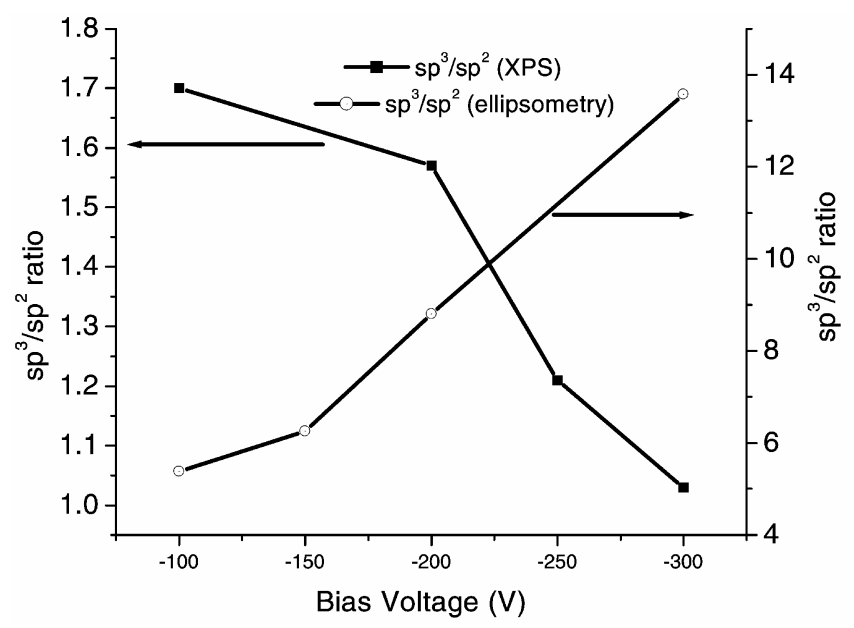

Figure 4. Comparative $s p^{3} / s p^{2}$ ratio as obtained by XPS and spectroscopic ellipsometry showing different trends in the $s p^{3} / s p^{2}$ ratio obtained by both the techniques (top)

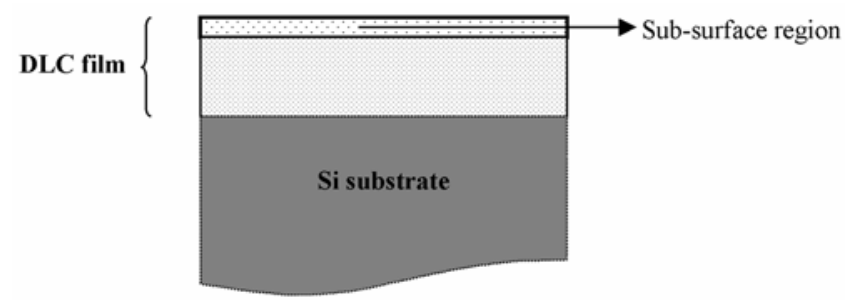

Figure 5. Two layered structure of DLC film showing a dense $s p^{3}$ rich layer under a low density $s p^{2}$ rich sub-surface region (bottom). Dimensions are not to be scaled. layers of the DLC network are compressed under high impact energy of incident ions, resulting in the staggered configuration of the $s p^{2}$ bonded aromatic rings. It may be noted that deviation from the planarity of the aromatic rings under compression results in a $\sigma-\pi$ mixing, responsible for the observed $s p^{3} / s p^{2}$ ratio for bulk of the sample. Ferrari and Robertson (2000) also suggested as to how the transformation in the six-fold carbon rings can affect the Raman spectrum in nano-crystalline (nc) graphite, particularly when its size and distribution in nc-graphite approaches that of molecular unit as in $a$-C. Bond angle distortions or bond bending are known to result in a staggered configuration, which in turn affect the $s p^{3} / s p^{2}$ ratio via $\sigma-\pi$ mixing.

\section{Conclusions}

Diamond like carbon films deposited by microwave CVD processes were studied using XPS and spectroscopic ellipsometry under bias voltage varying from $-100 \mathrm{~V}$ to $-300 \mathrm{~V}$. The $s p^{3} / s p^{2}$ ratio has been estimated by using both these techniques. Spectroscopic ellipsometry investigations have shown a composite layer of DLC film having two distinct layers with different void fractions, the bottom layer being more compact than the top layer. The $s p^{3} / s p^{2}$ ratio as determined by using spectroscopic ellipsometry is not in agreement with what is observed with XPS because the former technique investigates the entire DLC sample (sub-surface layer as well as interior layers), whereas the latter technique (XPS) investigates only the sub-surface layer. Therefore, investigations using spectroscopic ellipsometry are complimentary to those using XPS. Together, they provide a comprehensive picture of DLC growth.

\section{Acknowledgements}

Authors would like to thank Dr N Venkatramani and Dr S M Sharma for their constant encouragement. One of the authors would also like to thank Dr R D'Cunha for her valuable suggestions in carrying out these studies. (RMD) thanks PU-BARC, India for a fellowship and (SKK) thanks UGC, India, for continuous support.

\section{References}

Angus J C and Hayman C C 1988 Science 241913 Bruggeman D A 1935 Ann. Phys. (Leipzig) 24636 Ferrari A C and Robertson J 2000 Phys. Rev. B61 14095

Filik J, May P W, Pearce S R J, Wild R K and Hallam K R 2003 Dia. Relat. Mater. 12974

Forouhi A R and Bloomer I 1986 Phys. Rev. B34 7018

Grill A 2003 Dia. Relat. Mater. 12166

Kutsay O, Bello I, Lifshitz Y, Lam C W, Luk W Y, Lee S T, Meng X and Kremnican V 2003 Dia. Relat. Mater. 122051

Linder S, Wolfhard P and Aepfelbatier M 2002 Biomaterials 23 767 
Martin P J, Filipczuk S W, Netterfield R P, Field J S, Whitnal D F and Mckenzie D R 1988 J. Mater. Sci. Lett. 7410

Martinu L, Raveh A, Domingue A, Bertrand L, KlembergSapieha J E, Gujarathi S C and Wertheiner M R 1992 Thin Solid Films 20842

Martinu L, Raveh A, Boutard D, Houle S H, Poitras D, Vella N and Wertheimer M 1993 Dia. Relat. Mater. 2673

Pandey M, Bhattacharyya D, Patil D S, Ramachandran K and Venkatramani N 2004 Surf. Coat. Technol. 18224
Patil D S, Ramachandran K, Bhide A L and Venkatramani N 1997 Report: BARC/1997/E/025

Robertson J 1994 Pure \& Appl. Chem. 661789

Savvidas N 1986 J. Appl. Phys. 594133

Uhlmann S, Frauenheim Th and Lifshitz Y 1998 Phys. Rev. Lett. 81641

Weissmantel C, Bewilogua K, Dietrich D, Erler H J, Hinnerberg H, Klose S, Nowick W and Reisse G 1980 Thin Solid Films 7219 